\title{
Gender-related differences in the progression of carotid stiffness with age and in the influence of risk factors on carotid stiffness
}

This article was published in the following Dove Press journal:

Clinical Interventions in Aging

\author{
Maria Łoboz-Rudnicka' \\ Joanna Jaroch' \\ Ewa Kruszyńska' \\ Zbigniew Bociąga' \\ Barbara Rzyczkowska' \\ Krzysztof Dudek ${ }^{2}$ \\ Andrzej Szuba ${ }^{3}$ \\ Krystyna Łoboz-Grudzień ${ }^{1,4}$ \\ 'Department of Cardiology, \\ T. Marciniak Hospital, Wrocław, \\ Poland; ${ }^{2}$ Faculty of Mechanical \\ Engineering, Wrocław University of \\ Technology, Wrocław, Poland; ${ }^{3}$ Division \\ of Angiology, Wrocław Medical \\ University, Wrocław, Poland; ${ }^{4}$ Public \\ Health Department, Wrocław Medical \\ University, Wrocław, Poland
}

Correspondence: Maria Łoboz-Rudnicka Dolnoslaski Szpital Specjalistyczny im. T. Marciniaka, Department of Cardiology, ul. Fieldorfa 2, 54-049 Wrocław, Poland

Tel +48 7l 3064702

Fax +48713064875

Email marialoboz@o2.pl
Background: In recent years, there has been growing interest in the impact of gender-related factors on the function and structure of the arterial tree. The aim of our study was to identify gender-specific differences in the progression of carotid stiffness parameters with age and in the impact of risk factors on carotid stiffness.

Subjects and methods: The study group included 256 subjects (mean age: 54.7 years): 134 women $(52 \%)$ and 122 men (48\%) with cardiovascular risk factors: hypertension, type 2 diabetes mellitus, dyslipidemia, smoking, and obesity. Local parameters of carotid stiffness: $\beta$ stiffness index ( $\beta$ ), Peterson's elastic modulus (Ep), pulse wave velocity $\beta$ $(\mathrm{PWV}-\beta)$ and arterial compliance $(\mathrm{AC})$ were determined with ultrasound echo-tracking software application.

Results: Women were characterized by lower AC than men (women: $0.57 \mathrm{~mm}^{2} / \mathrm{kPa}$ vs men: $0.69 \mathrm{~mm}^{2} / \mathrm{kPa}, p<0.001$ ) and the subanalysis in three age groups revealed that the difference in $A C$ value between genders became significant over the age of 45 years. Although no significant difference in the value of $\beta$, Ep and PWV- $\beta$ were found between genders in the whole study group, women $<45$ years were characterized by lower values of $\beta$ and Ep than their men counterparts ( $\beta$ : women: 5.4 vs men: 6.6, $p=0.002$; Ep: women: $72 \mathrm{kPa}$ vs men: $84 \mathrm{kPa}, p=0.015$ ). Among analyzed risk factors, the significant determinants of carotid stiffness were age, blood pressure components (pulse pressure and mean arterial pressure), type 2 diabetes mellitus and heart rate. The relationship between carotid stiffness and pulse pressure was observed only in women and between carotid stiffness and heart rate - only in men.

Conclusion: There are gender-related differences in the progression of carotid stiffness parameters with age and in the influence of risk factors on carotid stiffness.

Keywords: arterial stiffness, gender, risk factors, arterial remodeling

\section{Introduction}

In recent years, there has been growing interest in the impact of gender-related factors on the function and structure of the arterial tree. Some evidence suggests that agerelated changes in the human vascular tree may follow different patterns in men and women and that the impact of risk factors on degenerative processes in the arterial wall might be modulated by gender.

Aside from atherosclerosis, stiffening is a major degenerative process that affects arterial wall properties. Adequate arterial compliance and distensibility result from a unique structure of elastin and collagen lamellae in the tunica media of the arterial wall and from correct endothelial function. Destruction and disorganization of the elastin lamellae, as well as endothelial dysfunction, lead to increased arterial stiffness, 
which has various unfavorable effects on the circulation. ${ }^{1}$ First of all, increased arterial stiffness impairs proper interaction between heart and arteries. Contributing to higher velocity of blood propagation in the aorta and faster return of reflected wave to the ascending aorta, arterial stiffness results in the rise of systolic blood pressure and decrease in diastolic blood pressure. This causes an increase in left ventricular afterload and inadequate blood supply of the myocardium, which may eventually lead to left ventricular hypertrophy, myocardial ischemia and heart failure. ${ }^{2,3}$ Moreover, increased arterial stiffness exerts deleterious effects within the microcirculation. Through transmission of pulsatile blood flow into capillaries, arterial stiffness contributes to progression of degenerative processes within the microcirculation, especially in the brain and kidneys. ${ }^{2}$ Various components are involved in the pathogenesis of arterial stiffening, among them, the mechanical impact of blood pressure on the arterial wall, metabolic disorders, and inflammation. A growing body of evidence suggests that inflammation plays a key role in the etiology of arterial stiffening and hence, a significant correlation may exist between inflammation markers and arterial stiffness parameters. ${ }^{4}$

The aim of this study was 1) to analyze age-related changes in local carotid stiffness parameters in men and women; 2) to study potential gender-specific differences in the progression of carotid stiffness indices with age; 3) to determine a relationship between cardiovascular risk factors and carotid stiffness in men and women; and 4) to verify if this relationship is modulated by gender.

\section{Subjects and methods}

The study included 256 subjects (mean age: 54.7 years): 134 women (52\%) and 122 men (48\%) with cardiovascular risk factors: hypertension, type 2 diabetes mellitus, dyslipidemia, smoking, and obesity. Exclusion criteria were: 1) history of a manifest cardiovascular disease (ischemic heart disease, stroke, lower limb atherosclerosis); 2) atheromatous plaques in carotid arteries on ultrasound; 3) electrocardiographic evidence of acute or remote myocardial ischemia; 4) echocardiographic evidence of regional left ventricular wall motion abnormalities, reduced left ventricle ejection fraction or valve disease. The study group was divided into three age groups: 1$)<45$ years $(n=52)$; 2) 45-60 years $(n=113)$; and 3$)>60$ years of age $(n=91)$.

The study subjects were considered hypertensive if: 1) they had been previously diagnosed with hypertension but did not receive a regular hypotensive treatment; or 2) were newly diagnosed with hypertension. Hypertension was diagnosed if on two separate visits (at least 7 days apart) the subjects presented with systolic blood pressure $\geq 140 \mathrm{mmHg}$ and/or diastolic blood pressure $\geq 90 \mathrm{mmHg}$ (after a 15-min rest).

Subjects were regarded as diabetic if they had a previous diagnosis of type 2 diabetes mellitus and received pharmacotherapy (oral agents or insulin).

Dyslipidemia was defined as follows: 1) low-density lipoprotein (LDL)-cholesterol fasting serum concentration $>115 \mathrm{mg} / \mathrm{dL}$; or 2) high-density lipoprotein (HDL)-cholesterol fasting serum concentration $<40 \mathrm{mg} / \mathrm{dL}$ in men and $<50 \mathrm{mg} / \mathrm{dL}$ in women; or 3) fasting triglyceride serum concentration $>150 \mathrm{mg} / \mathrm{dL}$; or 4) treatment with hypolipidemic agents.

Increased waist circumference was defined according to the European Society of Cardiology/European Society of Hypertension $(\mathrm{ESC} / \mathrm{ESH})$ guidelines as the circumference $\geq 88 \mathrm{~cm}$ in women and $\geq 102 \mathrm{~cm}$ in men. ${ }^{5}$

Overweight was defined as subjects with a BMI $\geq 25$ and $<30 \mathrm{~kg} / \mathrm{m}^{2}$, and obese was defined as subjects with a $\mathrm{BMI} \geq 30 \mathrm{~kg} / \mathrm{m}^{2}$.

The study participants were classified as active smokers if they were smoking cigarettes at the time of enrollment or had given up smoking within the previous 12 months.

Carotid stiffness parameters were calculated with the application of the echo-tracking (eT) system incorporated in Aloka ProSound Alpha 10 device (Figure 1). During the examination, the patient was in a relaxed, supine position while the ECG monitoring was performed. First, a clear longitudinal image of the walls of the right common carotid artery (Rt-CCA) was acquired with a linear transducer at frequencies $>7 \mathrm{MHz}$. Then, the eT gate for the measurement of arterial diameter changes across the cardiac cycle was positioned at the boundaries between the intima and media of the anterior and posterior wall of the Rt-CCA, $1-2 \mathrm{~cm}$ proximal to the bifurcation. Three to five beats were averaged to obtain a representative waveform. Finally, the value of blood pressure (measured on the brachial artery with the use of the certified OMRON sphygmomanometer) was entered into the system and the following carotid stiffness parameters were calculated automatically:

- B stiffness index $(\beta)$, as the ratio of the natural logarithm of systolic/diastolic blood pressure to the relative change in diameter:

$$
\beta=\ln (\mathrm{Ps} / \mathrm{Pd}) /[(\mathrm{Ds}-\mathrm{Dd}) / \mathrm{Dd}],
$$

where $\ln$, the natural logarithm; Ps, systolic blood pressure; $\mathrm{Pd}$, diastolic blood pressure; Ds, arterial systolic diameter; $\mathrm{Dd}$, arterial diastolic diameter. 


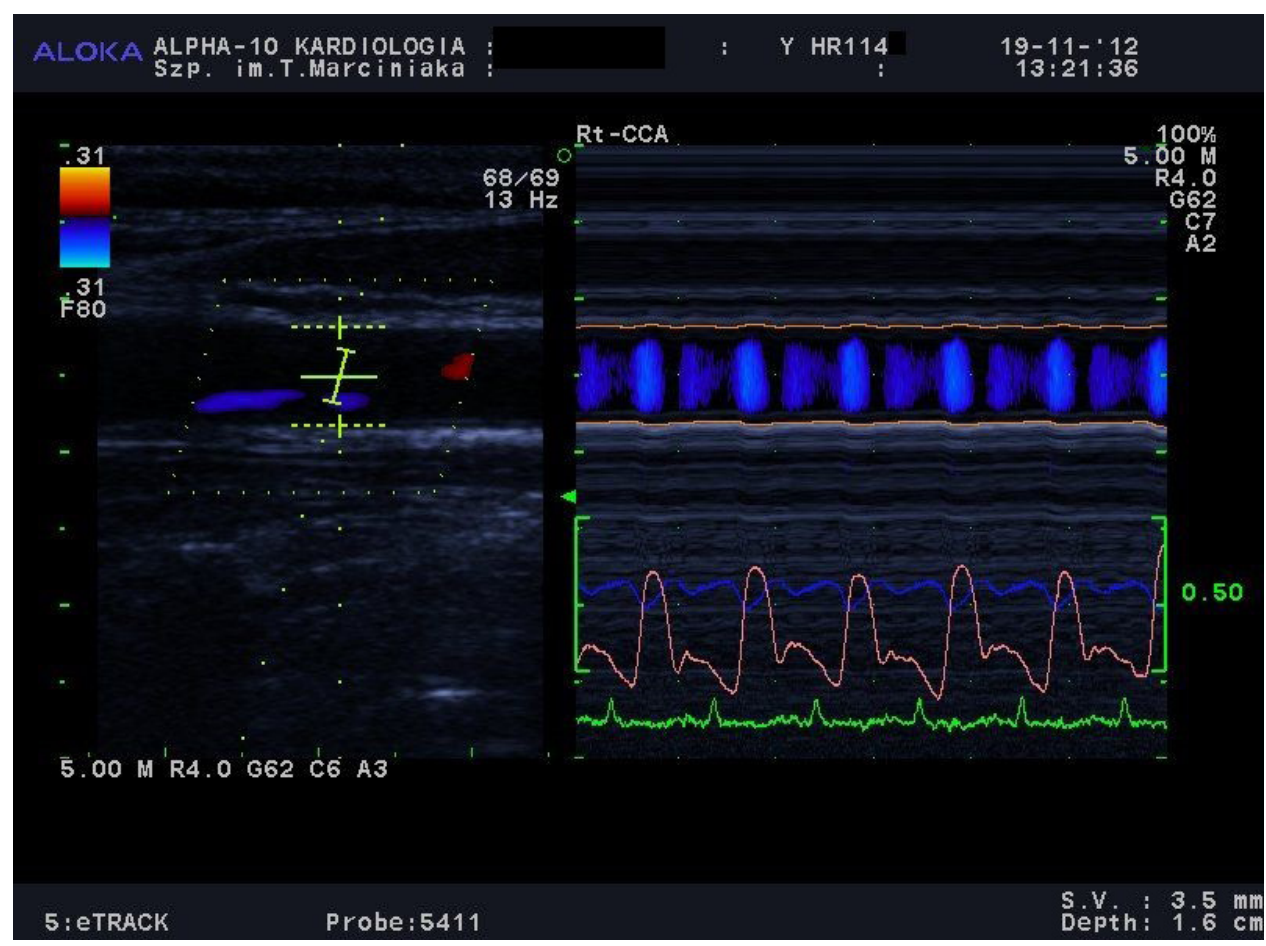

Figure I Assessment of the carotid stiffness-ultrasound echo-tracking application. Abbreviation: Rt-CCA, right common carotid artery.

- Peterson's elastic modulus (Ep):

$$
\mathrm{Ep}=(\mathrm{Ps}-\mathrm{Pd}) /[(\mathrm{Ds}-\mathrm{Dd}) / \mathrm{Dd}] .
$$

- Arterial compliance (AC), calculated from the arterial cross area and blood pressures:

$$
\mathrm{AC}=\mathrm{p}(\mathrm{Ds} \times \mathrm{Ds}-\mathrm{Dd} \times \mathrm{Dd}) /[4 \times(\mathrm{Ps}-\mathrm{Pd})] .
$$

- One-point pulse wave velocity $\beta$ (PWV- $\beta$ ), calculated from the time delay between two adjacent distension waveforms from the water hammer equation with the usage of $\beta$-stiffness parameter:

$$
P W V-\beta=\sqrt{ }(\beta \times P d / 2 \times \rho)
$$

where $\mathrm{Pd}$, diastolic blood pressure; $\rho$, blood density $\left(1,050 \mathrm{~kg} / \mathrm{m}^{3}\right)$.

All participants provided written informed consent. The study was approved by the Ethics Committee of Wrocław Medical University.

Statistical analysis was performed using STATISTICA Version 10 software and EXCEL spreadsheets. All quantitative parameters in all subgroups were tested for distribution normality using the Shapiro-Wilk test at a threshold significance level of $p<0.05$. Means (M), standard deviation (SD), median (Me) values, lower and upper quartiles (Q1 and Q3) or interquartile ranges (IQR), and extremes values: minimum (Min) and maximum (Max) were calculated for the quantitative parameters. For parameters with normal distributions and homogeneous variances, significance of differences between the means in two groups was verified using Student's $t$-test. For parameters with distributions significantly different from normal or heterogeneous variances, significance of differences between means in two groups was verified using the Mann-Whitney U-test, significance of differences between means in two dependent samples was verified using the Wilcoxon test, and significance of differences between means in more than two groups was verified using the Kruskal-Wallis test. For nominal variables (gender, presence of symptoms, etc.) numbers (n) and percentages (\%) were calculated and cross-tabulated. Hypotheses on the independence of nominal and ordinal characteristics were verified using Pearson's chi-squared test. For all tests, a significance threshold of $p<0.05$ was used.

\section{Results \\ Characteristics of the study group}

Characteristics of the study group are presented in Table 1. Mean age of the study subjects was $54.7 \pm 10.6$ years. Men and women did not differ significantly in terms of their age. Compared with men, women presented with lower values of: height, body weight, waist circumference, and waist-to-hip ratio. Moreover, women participants had significantly higher 
Table I Demographic and cardiovascular risk characteristics of the study group

\begin{tabular}{|c|c|c|c|c|}
\hline & $\begin{array}{l}\text { Total } \\
(n=256)\end{array}$ & $\begin{array}{l}\text { Women } \\
(n=134)\end{array}$ & $\begin{array}{l}\text { Men } \\
(n=I 22)\end{array}$ & $\begin{array}{l}\text { Women } \\
\text { vs men } \\
p\end{array}$ \\
\hline Age (years) & $54.7 \pm 10.6$ & $55.7 \pm 9.9$ & $53.5 \pm 11.3$ & 0.099 \\
\hline Height (cm) & $169 \pm 9$ & $162 \pm 5$ & $176 \pm 6$ & $<0.001$ \\
\hline Weight (kg) & $82 \pm 16$ & $74 \pm 13$ & $90 \pm 14$ & $<0.001$ \\
\hline BMI $\left(\mathrm{kg} / \mathrm{m}^{2}\right)$ & $28.6 \pm 4.5$ & $28.3 \pm 4.9$ & $29.0 \pm 3.9$ & 0.212 \\
\hline Waist circumference $(\mathrm{cm})$ & $95 \pm 14$ & $89 \pm 12$ & $103 \pm 11$ & $<0.001$ \\
\hline Hip circumference $(\mathrm{cm})$ & $108 \pm 9$ & $107 \pm 10$ & $108 \pm 8$ & 0.417 \\
\hline WHR & $0.89 \pm 0.09$ & $0.83 \pm 0.08$ & $0.95 \pm 0.07$ & $<0.001$ \\
\hline $\mathrm{HR}(/ \mathrm{min})$ & $70 \pm 10$ & $71 \pm 10$ & $69 \pm 9$ & 0.074 \\
\hline Hypertension & $160(65.8)$ & 78 (62.9) & $82(68.9)$ & 0.395 \\
\hline SBP $(\mathrm{mmHg})$ & $134 \pm 18$ & $133 \pm 19$ & $135 \pm 16$ & 0.474 \\
\hline $\mathrm{DBP}(\mathrm{mmHg})$ & $76 \pm 11$ & $77 \pm 11$ & $75 \pm 10$ & 0.147 \\
\hline MAP (mmHg) & $95 \pm 12$ & $96 \pm 12$ & $95 \pm 11$ & 0.602 \\
\hline $\mathrm{PP}(\mathrm{mmHg})$ & $58 \pm 14$ & $56 \pm 16$ & $60 \pm 12$ & 0.048 \\
\hline Diabetes mellitus type 2 & $90(35)$ & $40(30)$ & $50(40)$ & 0.118 \\
\hline Glucose* (mg/dL) & $101 \pm 23$ & $100 \pm 21$ & $103 \pm 26$ & 0.393 \\
\hline Dyslipidemia & $195(81.6)$ & $103(88.0)$ & $92(75.4)$ & 0.019 \\
\hline Total cholesterol* (mg/dL) & $220 \pm 4 I$ & $225 \pm 43$ & $213 \pm 37$ & 0.016 \\
\hline LDL-cholesterol* (mg/dL) & $130 \pm 39$ & $130 \pm 43$ & $129 \pm 34$ & 0.858 \\
\hline HDL-cholesterol* (mg/dL) & $54 \pm 16$ & $60 \pm 18$ & $49 \pm 12$ & $<0.001$ \\
\hline Triglycerides* (mg/dL) & $154 \pm 97$ & $150 \pm 80$ & $159 \pm 112$ & 0.505 \\
\hline Smokers & $94(36.7)$ & 45 (33.6) & $49(40.2)$ & 0.174 \\
\hline
\end{tabular}

Notes: *Fasting serum concentration. Values are mean \pm SD or number (\%). Statistically significant values are shown in bold.

Abbreviations: BMI, body mass index; WHR, waist hip ratio; HR, heart rate; SBP, systolic blood pressure; DBP, diastolic blood pressure; MAP, mean arterial pressure; PP, pulse pressure; LDL, low-density lipoprotein; HDL, high-density lipoprotein; SD, standard deviation.

fasting serum concentrations of total cholesterol and HDL cholesterol and more often satisfied the criteria of dyslipidemia. No significant differences were found in the prevalence of hypertension and type 2 diabetes mellitus among men and women, or in the smoking.

\section{Carotid stiffness parameters in men and women}

Median values of carotid stiffness parameters in men and women are presented in Table 2 and Figures 2-5. Regardless of gender, a significant increase in $\beta$ stiffness index, Ep and PWV- $\beta$ values was observed with participants' age, along with a significant decrease in AC. Noticeably, the most evident differences in median values of carotid stiffness parameters were observed between the first and second age group, either in women or in men.

The only stiffness parameter in which the median values differed significantly between men and women from the whole study group was AC, which turned out to be significantly lower in women study subjects. No significant gender-specific differences were found in median values of $\beta$ stiffness index, Ep and PWV- $\beta$ for the whole study group. However, some gender-related differences in the carotid stiffness parameters became evident after stratification of the study group according to age. Women from group 1 $(<45$ years of age) presented with significantly lower values of $\beta$ and Ep than men, whereas no statistically significant gender-specific differences in these parameters were observed in older age groups. In turn, women aged 45 years and older (group 2 and 3) showed significantly lower median values of $\mathrm{AC}$ than men from the same age categories, whereas no statistically significant differences in AC were found between men and women participants from group 1. Regardless of their age, men and women did not differ significantly in terms of median PWV- $\beta$ values.

\section{Risk factors}

The following risk factors were identified in the multivariate analysis as independent determinants of carotid stiffness parameters in the whole study group (Table 3 ): age (for all parameters), mean arterial pressure (for Ep, PWV- $\beta$ and AC), pulse pressure (for all parameters), type 2 diabetes mellitus (for $\beta$, Ep and PWV- $\beta$ ), heart rate (for Ep and PWV- $\beta$ ), and being a woman (only for AC). Whereas age, mean arterial pressure, and type 2 diabetes mellitus influenced carotid stiffness parameters in both men and women, pulse pressure was related to stiffness parameters $(\beta$, Ep and PWV- $\beta$ ) in women subjects only (Table 4). Heart rate, 


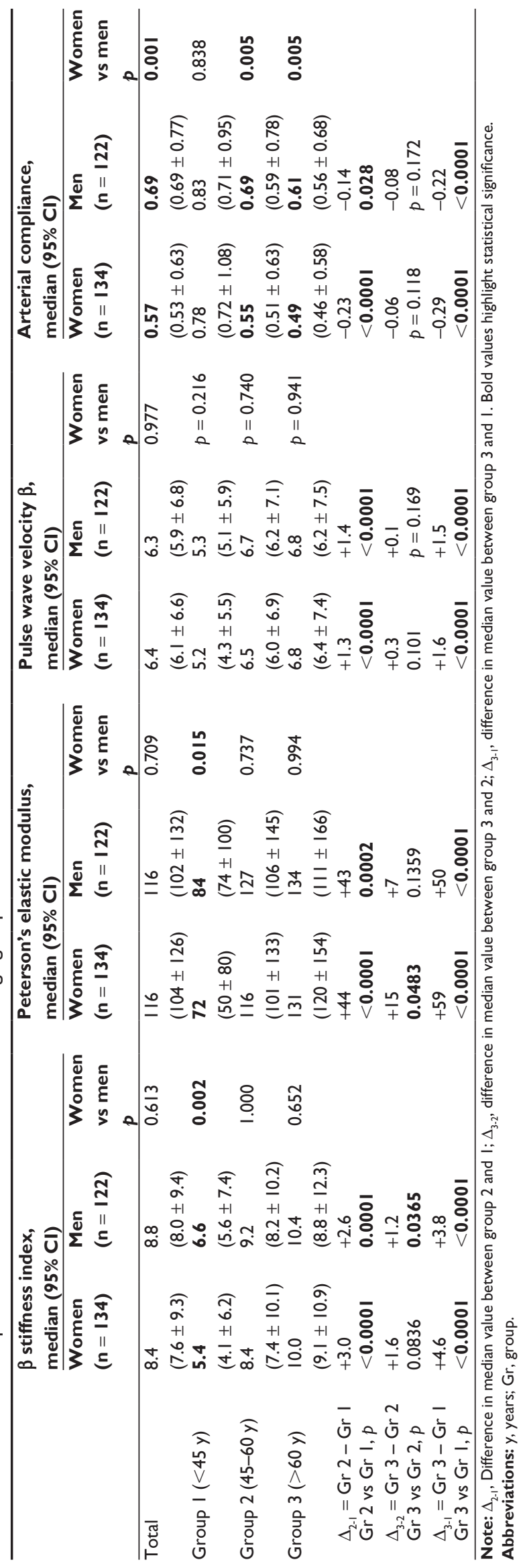

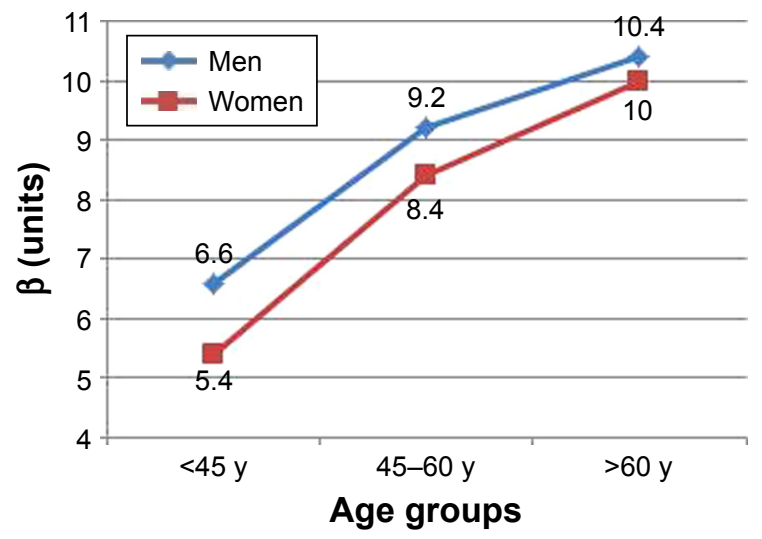

Figure 2 Median $\beta$ stiffness index $(\beta)$ in three age groups in men and women. Abbreviation: $y$, years.

which was a determinant of stiffness parameters (Ep and PWV- $\beta$ ) in the whole study group, was not a significant factor in the subanalysis of men and women. However, it is worth mentioning that in the univariate analysis, heart rate was related to stiffness parameters (Ep and PWV- $\beta$ ) in men subjects.

\section{Discussion}

Our study demonstrated gender-specific differences in the progression of carotid stiffness parameters with age, and showed that patient gender modulated the association between risk factors and carotid stiffness. Our findings provide new insight into the relationship between gender and progression of arterial stiffness - an issue which is still a subject of ongoing research.

Many previous studies have shown that age is the major determinant of arterial stiffness. ${ }^{6-8}$ Our findings are consistent with those observations. In both men and women median values of $\beta, E p$, and PWV- $\beta$ increased with age, whereas AC decreased. The most evident differences in carotid stiffness

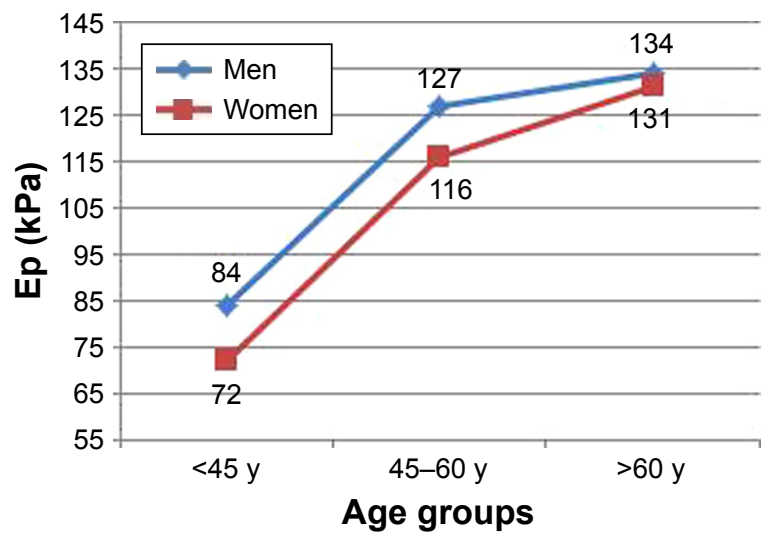

Figure 3 Median Peterson's elastic modulus in three age groups in men and women. Abbreviations: Ep, Peterson's elastic modulus; $y$, years. 


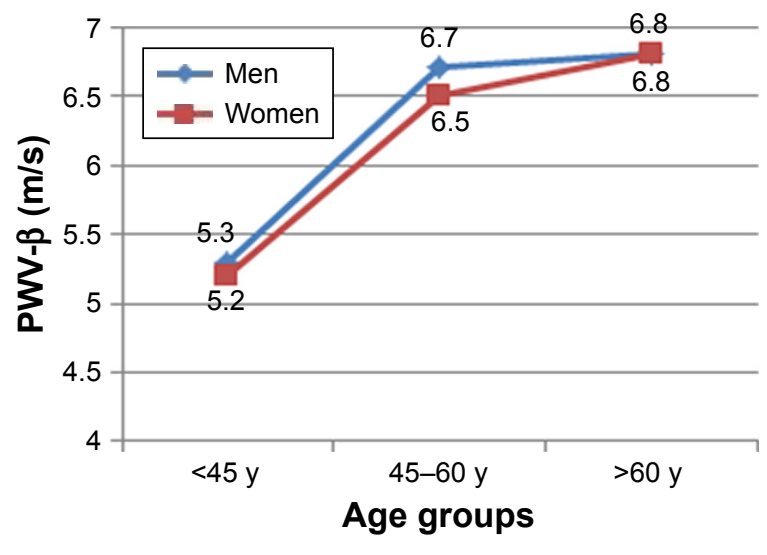

Figure 4 Median PWV $\beta$ in three age groups in men and women. Abbreviations: PWV, pulse wave velocity; $y$, years.

parameters were observed between middle-aged (45-60 years) and young ( $<45$ years $)$ study subjects. Similar results were reported previously from Framingham Heart Study offspring cohort and from Anglo-Cardiff Collaborative Trial - both those studies demonstrated that the age-related increase in central arterial stiffness was not linear, but progressed slowly in younger persons, and then accelerated in those older than 50 years. ${ }^{6,7}$

A primary aim of our study was to compare the patterns of age-related changes in carotid stiffness in men and women. Analysis of the whole study group showed that women presented with lower AC than men. However, when the study subjects were stratified according to their age, the difference in median AC in men and women reached the threshold of statistical significance solely among subjects older than 45 years. In turn, no statistically significant gender-specific differences in median values of $\beta$ and Ep were found for the whole study group, but women patients younger than 45 years presented with significantly lower values of those parameters than men participants from the same age category.

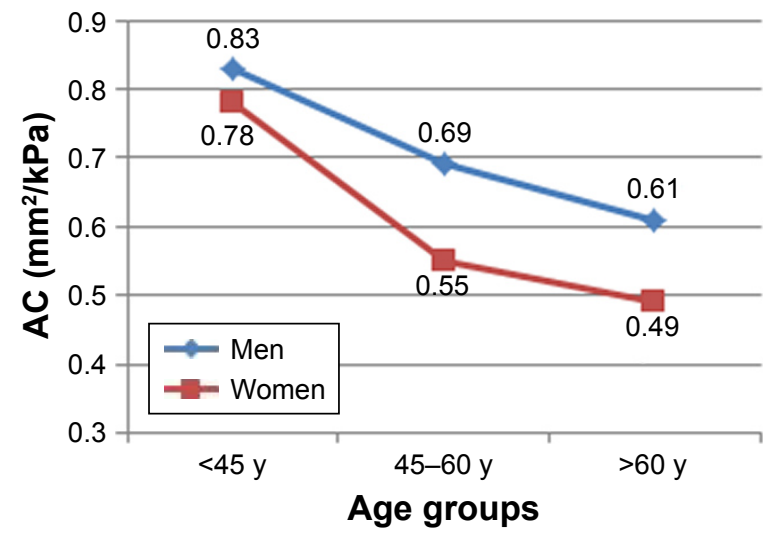

Figure $\mathbf{5}$ Median $A C$ in men and women in three age groups. Abbreviations: AC, arterial compliance; $y$, years.
Table 3 Multivariate analysis: relationship between cardiovascular risk factors and carotid stiffness parameters in the whole study group

\begin{tabular}{|c|c|c|c|c|}
\hline & $\begin{array}{l}\beta \\
\text { stiffness } \\
\text { index }\end{array}$ & $\begin{array}{l}\text { Peterson's } \\
\text { elastic } \\
\text { modulus }\end{array}$ & $\begin{array}{l}\text { Pulse wave } \\
\text { velocity } \beta\end{array}$ & $\begin{array}{l}\text { Arterial } \\
\text { compliance }\end{array}$ \\
\hline & $p$ & $p$ & $p$ & $p$ \\
\hline Age & $<0.001$ & $<0.001$ & $<0.001$ & $<0.001$ \\
\hline DM2 & $<0.00 \mathrm{I}$ & 0.001 & $<\mathbf{0 . 0 0 1}$ & $x$ \\
\hline PP & $<0.00$ I & $<\mathbf{0 . 0 0 I}$ & 0.001 & 0.001 \\
\hline MAP & $x$ & 0.001 & $<\mathbf{0 . 0 0 1}$ & $<0.05$ \\
\hline Heart rate & $x$ & $<0.05$ & $<0.05$ & 0.7 \\
\hline Hypertension & 0.8 & 0.9 & 0.8 & $x$ \\
\hline Being a woman & $x$ & $x$ & $x$ & 0.001 \\
\hline
\end{tabular}

Notes: ' $x$ ' indicates not included in the model. Statistically significant values are shown in bold.

Abbreviations: DM2, diabetes mellitus type 2; PP, pulse pressure; MAP, mean arterial pressure.

The observation that arterial stiffness in women was lower than in men only in the premenopausal period is consistent with the results published by some other authors. In a Korean study, arterial stiffness, determined based on cardio-ankle vascular index (CAVI) values, turned out to be significantly lower in 30- to 59-year-old women than in men from the same age group - but no gender-specific differences in CAVI values were observed in subjects older than 60 years. ${ }^{9}$ A similar pattern of age-related changes in aortic stiffness parameters was also documented in an Australian population: younger women presented with lesser aortic stiffness than younger men, but due to a more dynamic increase in this parameter during the perimenopausal period aortic stiffness in older women turned out to be significantly higher than in older men. ${ }^{10}$ However, contradictive results were obtained in The Baltimore Longitudinal Study of Aging, a prospective study conducted in an American population of subjects without cardiovascular disease; in that study men showed more evident increase in carotid-femoral pulse wave velocity (cf-PWV) and presented higher values of cf-PWV than women, especially after 50 years of age. ${ }^{11}$ Those discrepancies might result from the fact that the abovementioned studies were performed in different populations, analyzed various parameters of arterial stiffness and had different design. Consequently, further research is needed to verify how gender influences progression of arterial stiffness with age.

\section{Blood pressure components}

Aside from age, other significant determinants of carotid stiffness are the components of blood pressure: mean arterial pressure and pulse pressure. The relationship between arterial stiffness and blood pressure seems to be bidirectional. On the one hand, elevated systolic blood pressure is associated with 
Table 4 Multivariate analysis: relationship between cardiovascular risk factors and carotid stiffness parameters in men and women

\begin{tabular}{|c|c|c|c|c|c|c|c|c|}
\hline & \multicolumn{2}{|c|}{$\beta$ stiffness index } & \multicolumn{2}{|c|}{$\begin{array}{l}\text { Peterson's elastic } \\
\text { modulus }\end{array}$} & \multicolumn{2}{|c|}{ Pulse wave velocity $\beta$} & \multicolumn{2}{|c|}{ Arterial compliance } \\
\hline & Women & Men & Women & Men & Women & Men & Women & Men \\
\hline & $p$ & $p$ & $p$ & $p$ & $p$ & $p$ & $p$ & $p$ \\
\hline Age & $<0.001$ & $<0.001$ & 0.004 & $<0.001$ & $<\mathbf{0 . 0 0 1}$ & $<\mathbf{0 . 0 0 1}$ & $<\mathbf{0 . 0 0 1}$ & $<0.00$ I \\
\hline DM2 & 0.003 & 0.003 & $<0.05$ & 0.003 & 0.005 & 0.002 & $x$ & $x$ \\
\hline PP & 0.006 & 0.2 & $<\mathbf{0 . 0 0 1}$ & 0.09 & 0.007 & 0.01 & 0.08 & $x$ \\
\hline MAP & $x$ & 0.2 & 0.2 & $<0.001$ & 0.01 & $<0.001$ & 0.002 & 0.003 \\
\hline Heart rate & $x$ & 0.09 & $x$ & 0.1 & $x$ & 0.01 & $x$ & 0.2 \\
\hline Hypertension & $x$ & $x$ & 0.5 & $x$ & 0.5 & $x$ & $x$ & $x$ \\
\hline
\end{tabular}

Notes: ' $x$ ' indicates not included in the model. Statistically significant values are shown in bold.

Abbreviations: DM2, diabetes mellitus type 2; PP, pulse pressure; MAP, mean arterial pressure.

greater shear stress acting on the arterial wall, which results in faster progression of arterial stiffness. On the other hand, increased arterial stiffness leads to faster propagation of the forward pulse wave and earlier return of the reflected wave to the ascending aorta during the systole; this results in an increase in systolic blood pressure, decrease in diastolic blood pressure, and ultimately higher pulse pressure.

In our study, both mean arterial pressure and pulse pressure were significantly associated with carotid stiffness parameters. Literature provides a large body of evidence for the significant relationship between mean arterial pressure and various arterial stiffness parameters. ${ }^{12-15}$ However, some studies indicate that it is pulse pressure, which shows the strongest correlation with arterial stiffness parameters of all blood pressure components. In an invasive study conducted in a Korean population of normotensives and untreated hypertensives, correlation between pulse pressure and aortofemoral PWV turned out to be stronger than associations between mean arterial pressure or systolic blood pressure and aorto-femoral PWV. ${ }^{16}$

Published evidence regarding gender-specific differences in the relationship between blood pressure components and arterial stiffness is sparse. In our study, mean arterial pressure exerted a similar effect on carotid stiffness in men and women, but the relationship between pulse pressure and carotid stiffness was observed solely in women. A significant contribution of pulse pressure to arterial stiffness in women was also reported by Weinberger et al who examined a group of normo- and hypertensives. ${ }^{17}$ Among normotensives, a relationship between pulse pressure and small artery oscillatory elasticity was significant solely in women, and in hypertensives, both in women and men, but still stronger in the former. ${ }^{17}$

\section{Diabetes mellitus}

Type 2 diabetes mellitus is an established determinant of increased arterial stiffness. However, it is still unclear, whether the effect of diabetes on the development and progression of arterial stiffness is gender-specific. In our study, the relationship between type 2 diabetes mellitus and carotid stiffness parameters was statistically significant in both men and women. However, there are studies that show a gender-specific influence of diabetes on arterial stiffness. De Angelis demonstrated that type 2 diabetes mellitus accelerated age-related increase in arterial stiffness solely in women. ${ }^{18}$ To eliminate the potential confounding effect of hypertension on arterial stiffness, diabetic and non-diabetic participants of that study were matched for mean arterial pressure. ${ }^{18}$ Also, in the ARIC study serum concentrations of glucose and insulin exerted stronger effects on arterial stiffness parameters in women than in men. ${ }^{19} \mathrm{~A}$ tendency to greater arterial stiffness in women than in men was also observed among patients with type 1 diabetes. ${ }^{20}$ The underlying mechanisms of greater arterial stiffness in diabetic women are yet to be fully understood, but potential explanation is the loss of the beneficial effect of estrogens on the arterial tree due to disorders of glucose metabolism.

\section{Heart rate}

A relationship between heart rate and arterial stiffness may be explained by several mechanisms. First, faster heart rate accounts for a greater number of cardiac cycles over time, which results in higher sheer stress exerted on the arterial tree and thus, in faster degradation of elastin within the arterial wall. ${ }^{21}$ Further, increased heart rate may be a consequence of enhanced adrenergic tone, and adrenergic stimulation may contribute to greater arterial stiffness via various mechanisms, such as increased muscular tone and induction of elastin degradation within the arterial wall. ${ }^{22,23}$

Our study demonstrated that heart rate was an independent determinant of carotid stiffness. This observation is consistent with the results of previous studies. ${ }^{24}$ While in the multivariate analysis we found no gender-specific differences in the influence of heart rate on arterial stiffness, 
in the univariate analysis, the link between heart rate and carotid stiffness turned out to be stronger in men than in women. This finding seems to be consistent with the results published by other authors. Both in the SAPALDIA study (in which carotid stiffness parameters were examined) and in the Whitehall II study (in which cf-PWV was examined) heart rate was identified as a more powerful determinant of arterial stiffness in men than in women. ${ }^{25,26}$

\section{Lipids, obesity, and smoking}

Published data regarding a relationship between lipids, obesity, smoking, and arterial stiffness are inconclusive. Many studies, including our present research, did not find a significant relationship between these factors and parameters of arterial stiffness. ${ }^{12,15,16,18,27}$ However, some authors have reported contradictive results. In a Brazilian study, triglycerides turned out to be a significant determinant of both cf-PWV and local carotid stiffness, and in a Swiss SAPALDIA study significant relationships were observed between local carotid stiffness and: triglycerides in women, LDL-cholesterol in men, and HDL cholesterol in both genders. ${ }^{25,28}$ The same SAPALDIA study demonstrated a significant link between obesity and carotid stiffness in both men and women. A significant effect of BMI on arterial stiffness was also observed in a population of young women. ${ }^{29}$ Finally, both the SAPALDIA study and The Anglo-Cardiff Collaborative Trial identified smoking as a predictor of increased arterial stiffness. ${ }^{25,30}$

As a result of the abovementioned discrepancies, the relationship between factors such as dyslipidemia, obesity, smoking, and arterial stiffness needs to be verified in future studies.

\section{Limitations}

Overall, 15.9\% of subjects with dyslipidemia received statins, which might affect a relationship between cholesterol metabolism and carotid stiffness. Analyzing a link between smoking and carotid stiffness, we did not consider the duration of smoking and the number of cigarettes per day. Furthermore, we did not analyze the influence of inflammatory markers, nutritional factors, physical activity and psychosocial factors on carotid stiffness.

\section{Conclusion}

The results of this study imply that some gender-specific differences may exist in the progression of arterial stiffness with age. Specifically, we demonstrated that young women presented with less arterial stiffness than young men, but this beneficial effect of being a woman was no longer observed at the perimenopausal age. Furthermore, the interaction between some risk factors and arterial stiffness might be modulated by gender: with a stronger relationship between stiffness and pulse pressure in women and heart rate in men. So far, published data on gender-specific differences in the impact of various risk factors on progression of arterial stiffness are limited and inconclusive, which justifies further research in this area.

\section{Disclosure}

The authors report no conflicts of interest in this work.

\section{References}

1. Kohn JC, Lampi MC, Reinhart-King CA. Age-related vascular stiffening: causes and consequences. Front Genet. 2015;6:112.

2. O'Rourke MF. Arterial aging: pathophysiological principles. Vasc Med. 2007;12(4):329-341.

3. Antonini-Canterin F, Carerj S, Di Bello V, et al; Research Group of the Italian Society of Cardiovascular Echography (SIEC). Arterial stiffness and ventricular stiffness: a couple of diseases or a coupling disease? A review from the cardiologist's point of view. Eur JEchocardiogr. 2009; 10(1):36-43.

4. Mozos I, Malainer C, Horbańczuk J, et al. Inflammatory markers for arterial stiffness in cardiovascular diseases. Front Immunol. 2017;8: 1058.

5. Mancia G, Fagard R, Narkiewicz K, et al. 2013 ESH/ESC guidelines for the management of arterial hypertension: the Task Force for the Management of Arterial Hypertension of the European Society of Hypertension (ESH) and of the European Society of Cardiology (ESC). Eur Heart J. 2013;34(28):2159-2219.

6. McEniery CM, Yasmin, Hall IR, Qasem A, Wilkinson IB, Cockcroft JR; ACCT Investigators. Normal vascular aging: differential effects on wave reflection and aortic pulse wave velocity: the Anglo-Cardiff Collaborative Trial (ACCT). J Am Coll Cardiol. 2005;46(9):1753-1760.

7. Mitchell GF, Parise H, Benjamin EJ, et al. Changes in arterial stiffness and wave reflection with advancing age in healthy men and women: the Framingham Heart Study. Hypertension. 2004;43(6):1239-1245.

8. Mattace-Raso F, Hofman A, Verwoert GC, et al. Determinants of pulse wave velocity in healthy people and in the presence of cardiovascular risk factors: 'establishing normal and reference values'. Eur Heart $J$. 2010;31(19):2338-2350.

9. Choi SY, Oh BH, Bae Park J, Choi DJ, Rhee MY, Park S. Ageassociated increase in arterial stiffness measured according to the cardio-ankle vascular index without blood pressure changes in healthy adults. J Atheroscler Thromb. 2013;20(12):911-923.

10. Waddell TK, Dart AM, Gatzka CD, Cameron JD, Kingwell BA. Women exhibit a greater age-related increase in proximal aortic stiffness than men. J Hypertens. 2001;19(12):2205-2212.

11. Al Ghatrif M, Strait JB, Morrell CH, et al. Longitudinal trajectories of arterial stiffness and the role of blood pressure: the Baltimore Longitudinal Study of Aging. Hypertension. 2013;62(5):934-941.

12. Alecu C, Gueguen R, Aubry C, et al. Determinants of arterial stiffness in an apparently healthy population over 60 years. J Hum Hypertens. 2006;20(10):749-756.

13. McIntyre NJ, Fluck RJ, McIntyre CW, Fakis A, Taal MW. Determinants of arterial stiffness in chronic kidney disease stage 3. PLoS One. 2013;8(1):e55444.

14. Protogerou AD, Blacher J, Aslangul E, et al. Gender influence on metabolic syndrome's effects on arterial stiffness and pressure wave reflections in treated hypertensive subjects. Atherosclerosis. 2007;193(1): $151-158$. 
15. Paini A, Boutouyrie P, Calvet D, Tropeano AI, Laloux B, Laurent S. Carotid and aortic stiffness: determinants of discrepancies. Hypertension. 2006;47(3):371-376.

16. Kim EJ, Park CG, Park JS, et al. Relationship between blood pressure parameters and pulse wave velocity in normotensive and hypertensive subjects: invasive study. J Hum Hypertens. 2007;21(2):141-148.

17. Weinberger MH, Fineberg NS, Fineberg SE. Effects of age, race, gender, blood pressure, and estrogen on arterial compliance. Am J Hypertens. 2002;15(4 Pt 1):358-363.

18. De Angelis L, Millasseau SC, Smith A, et al. Sex differences in age-related stiffening of the aorta in subjects with type 2 diabetes. Hypertension. 2004;44(1):67-71.

19. Salomaa V, Riley W, Kark JD, Nardo C, Folsom AR. Non-insulindependent diabetes mellitus and fasting glucose and insulin concentrations are associated with arterial stiffness indexes. The ARIC Study. Atherosclerosis Risk in Communities Study. Circulation. 1995;91(5): $1432-1443$.

20. Ahlgren AR, Astrand H, Sundkvist G, Länne T. Increased aortic stiffness is persistent in type 1 diabetic women: a follow-up study. Diabetologia. 2005;48(4):780-783.

21. Palatini P, Julius S. Heart rate and the cardiovascular risk. J Hypertens. 1997;15(1):3-17.

22. Zieman SJ, Melenovsky V, Kass DA. Mechanisms, pathophysiology, and therapy of arterial stiffness. Arterioscler Thromb Vasc Biol. 2005; 25(5):932-943.

23. Aroor AR, Demarco VG, Jia G, et al. The role of tissue renin-angiotensinaldosterone system in the development of endothelial dysfunction and arterial stiffness. Front Endocrinol (Lausanne). 2013;4:161.
24. Benetos A, Adamopoulos C, Bureau JM, et al. Determinants of accelerated progression of arterial stiffness in normotensive subjects and in treated hypertensive subjects over a 6-year period. Circulation. 2002; 105(10):1202-1207.

25. Caviezel S, Dratva J, Schaffner E, et al. Sex-specific associations of cardiovascular risk factors with carotid stiffness - results from the SAPALDIA cohort study. Atherosclerosis. 2014;235(2):576-584.

26. Johansen NB, Vistisen D, Brunner EJ, et al. Determinants of aortic stiffness: 16-year follow-up of the Whitehall II study. PLoS One. 2012; 7(5):e37165.

27. Amar J, Ruidavets JB, Chamontin B, Drouet L, Ferrières J. Arterial stiffness and cardiovascular risk factors in a population-based study. J Hypertens. 2001;19(3):381-387.

28. Tolezani EC, Costa-Hong V, Correia G, Mansur AJ, Drager LF, Bortolotto LA. Determinants of functional and structural properties of large arteries in healthy individuals. Arq Bras Cardiol. 2014;103(5): 426-432.

29. Mizia-Stec K, Gasior Z, Zahorska-Markiewicz B, et al. The indexes of arterial structure and function in women with simple obesity: a preliminary study. Heart Vessels. 2008;23(4):224-229.

30. McEniery CM, Yasmin, Maki-Petaja KM, et al; Anglo-Cardiff Collaboration Trial Investigators. The impact of cardiovascular risk factors on aortic stiffness and wave reflections depends on age: the Anglo-Cardiff Collaborative Trial (ACCT III). Hypertension. 2010;56(4):591-597.
Clinical Interventions in Aging

\section{Publish your work in this journal}

Clinical Interventions in Aging is an international, peer-reviewed journal focusing on evidence-based reports on the value or lack thereof of treatments intended to prevent or delay the onset of maladaptive correlates of aging in human beings. This journal is indexed on PubMed Central, MedLine,

\section{Dovepress}

CAS, Scopus and the Elsevier Bibliographic databases. The manuscript management system is completely online and includes a very quick and fair peer-review system, which is all easy to use. Visit http://www.dovepress. com/testimonials.php to read real quotes from published authors. 